\title{
Correction to: National propensities?
}

\author{
David Mills $^{1} \mathbb{D} \cdot$ Kirsten Bell $^{2}$
}

C Akadémiai Kiadó, Budapest, Hungary 2021

\section{Correction to: Scientometrics https://doi.org/10.1007/s11192-021-04014-w}

In the original publication of the article the author name "Teixeira da Silva, J.A." was incorrectly published. This has been corrected with this Correction.

The original article has been corrected.

\section{References}

Tsigaris, P., \& Teixeira da Silva, J. A. (2020). Reproducibility issues with correlating Beall-listed publications and research awards at a small Canadian business school. Scientometrics, 123(1), 143-157. https://doi.org/10.1007/s11192-020-03353-4.

The original article can be found online at https://doi.org/10.1007/s11192-021-04014-w.

David Mills

david.mills@education.ox.ac.uk

1 Department of Education, University of Oxford, Oxford, UK

2 Department of Life Sciences, University of Roehampton, London, UK 